\title{
FRACTIONAL ORDER THEORY OF THERMAL STRESSES IN A TWO DIMENSIONAL TRANSVERSELY ISOTROPIC MAGNETO THERMOELASTIC MATERIAL
}

\author{
PARVEEN LATA, IQBAL KAUR* \\ Department of Basic and Applied Sciences, Punjabi University, Patiala, \\ Punjab, India
}

[Received: 29 July 2019. Accepted: 03 February 2020]

doi: 10.7546/JTAM.50.20.03.02

\begin{abstract}
A mathematical model for homogeneous transversely isotropic magneto-thermoelastic (HTIMT) material and fractional order theory (FOT) of thermal stresses due to ramp type heat and with hall current effect is formulated. To solve the field equations, Fourier and Laplace transforms are used. The mathematical expressions of components of displacement, temperature, current density and stress are solved in the transformed domain and then obtained in physical domain using transform inversion techniques. The fractional order parameter by considering various cases of weak, normal and strong conductivity are represented graphically.
\end{abstract}

KEY WORDS: Fractional-order Theory, Hall current, Magneto Thermoelastic, Transversely Isotropic, Ramp Type Heat.

\section{NOMENCLATURE}

$\delta_{i j} \quad$ : Kronecker delta,

$\vec{H}_{0} \quad$ : magnetic field intensity vector,

$\tau_{v}:$ : temperature gradient phase lag,

$T$ : temperature,

$c_{i j k l}:$ elastic parameters,

$\rho \quad$ : medium density,

$\varepsilon_{0} \quad$ : electric permeability,

$\tau_{t} \quad$ : heat flux phase lag,

$K_{i j}^{*}$ : materialistic constant,

$C_{E}$ : specific heat,

$e_{i j}$ : strain tensors,

$F_{i}$ : components of Lorentz force,

$T_{0}$ : reference temperature,

$\vec{j} \quad$ : current density vector,

$t_{i j}:$ stress tensors,

$\mu_{0} \quad$ : magnetic permeability,

$\vec{u} \quad$ : displacement vector,

$\tau_{0} \quad$ : relaxation time,

$u_{i}$ : components of displacement,

$\varphi$ : conductive temperature,

$\omega$ : frequency,

$\beta_{i j}:$ thermal elastic coupling tensor,

$K_{i j}$ : thermal conductivity,

$\tau_{q}$ : phase lag of thermal displacement,

$\alpha_{i j}$ : linear thermal expansion coefficient,

$a_{i j}:$ two temperature parameters,

$\delta(t)$ : Dirac's delta function,

$\alpha \quad$ : fractional parameter

$t_{0}:$ rise time of heat

$T_{1}$ : constant temperature

$T_{i j}$ : Maxwell's stress component

\footnotetext{
${ }^{*}$ Corresponding author e-mail: bawahanda@gmail.com
} 


\section{INTRODUCTION}

In last decade, the attraction of ample number of researchers for magneto-thermoelastic (MT) material (composite material) structures is continuously increasing since magnetothermoelastic materials exhibits the prerequisite coupling effect among electric and magnetic fields. Due to these electric and magnetic field, Hall voltage is produced in the medium. Similarly, studying the coupling effect in a thermoelastic medium among magnetic fields and stress is known as Magneto-thermoelasticity and has a numerous applications in the domain of science and technology mainly in nuclear reactors, geophysics, and related domains.

Sherief et al. [1] proposed a model of thermoelasticity by using fractional calculus and Youseff $[2,3]$ offered an alternative theory of thermoelasticity by using the fractional calculus with extensive range $(0<\alpha \leq 2)$. Ezzat et al. [4-6] established a additional theory on FOT using Taylor series. Bachher et al. [7] examined the Caputo time-FOT for MT response of a 2D isotropic solid including rotating. Kumar et al. [8] examined the FOT proposed by Sherief [1] and Ezzat \& Youssef [2] in a micropolar solid and ramp heat source. Also, Kumar et al. [9] studied the thermomechanical exchanges in a rotating transversely isotropic thermoelastic (TIT) solid with two temperatures (2T). Sheoran et al. [10] analysed the future predictions of FOT. Abbas [11] discussed 2-D GN-III model with FOT. Additionally, other researcher discussed another theories of thermoelasticity as Marin [12,13], Marin et al. [14,15], Lata and Kaur [16,17], Marin [18,19], Kumar et al. [20] Lata and Kaur [21-23], Lata \& Kaur [24-26], Marin and Craciun [27], Kaur and Lata [28]. Numerous research is done by researchers to solve different problem using fraction order generalized thermoelasticity. Regardless of these, no considerable research to study fractional order three phase lag (TPL) effect due to Hall current has been taken. In this research, a mathematical model is purposed to study the effect of FOT with hall current and ramp type heat in HTIMT solid.

\section{BASIC EQUATIONS}

The Maxwell's stress components and simplified linear equations for slow moving conducting elastic solid following [29] are:

$$
\begin{gathered}
\operatorname{curl} \vec{h}=\vec{j}+\varepsilon_{0} \frac{\partial \vec{E}}{\partial t}, \\
\operatorname{curl} \vec{E}=-\mu_{0} \frac{\partial \vec{h}}{\partial t}, \\
\vec{E}=-\mu_{0}\left(\frac{\partial \vec{u}}{\partial t}+\vec{H}_{0}\right),
\end{gathered}
$$




$$
\begin{gathered}
\operatorname{div} \vec{h}=0 . \\
T_{i j}=\mu_{0}\left(H_{i} h_{j}+H_{j} h_{i}-H_{k} h_{k} \delta_{i j}\right) .
\end{gathered}
$$

The equation of motion [30] for a TITM with the Lorentz force $F_{i}=\mu_{0}\left(\vec{j} \times \vec{H}_{0}\right)$ is given as under:

$$
\begin{gathered}
t_{i j}=c_{i j k l} e_{k l}-\beta_{i j} T, \\
t_{i j, j}+F_{i}=\rho \ddot{u}_{i} .
\end{gathered}
$$

The equations (6) and (7) using the universal Ohm's law with Hall current and with constant conductivity is given by

$$
J=\frac{\sigma_{0}}{1+m^{2}}\left(E+\mu_{0}\left(\dot{u} \times H-\frac{1}{e n_{e}} J \times H_{0}\right)\right) .
$$

The TPL heat conduction equation with FOT is

(9) $\quad K_{i j}\left(1+\frac{\left(\tau_{t}\right)^{\alpha}}{\alpha !} \frac{\partial^{\alpha}}{\partial t^{\alpha}}\right) \dot{T}_{, j i}+K_{i j}^{*}\left(1+\frac{\left(\tau_{v}\right)^{\alpha}}{\alpha !} \frac{\partial^{\alpha}}{\partial t^{\alpha}}\right) T_{, j i}$

$$
=\left(1+\frac{\left(\tau_{q}\right)^{\alpha}}{\alpha !} \frac{\partial^{\alpha}}{\partial t^{\alpha}}+\frac{\left(\tau_{q}\right)^{2 \alpha}}{2 \alpha !} \frac{\partial^{2 \alpha}}{\partial t^{2 \alpha}}\right)\left[\rho C_{E} \ddot{T}+\beta_{i j} T_{0} \ddot{e}_{i j}\right],
$$

where

$$
\beta_{i j}=c_{i j k l} \alpha_{i j}, \quad e_{i j}=\frac{1}{2}\left(u_{i, j}+u_{j, i}\right), \quad \beta_{i j}=\beta_{i} \delta_{i j}, \quad K_{i j}=K_{i} \delta_{i j},
$$

where $i, j=1,2,3$ and $i, j$ is not summed. Here $c_{i j k l}=c_{k l i j}=c_{j i k l}=c_{i j l k}$.

\section{Formulation and Solution of the Problem}

We consider a HTIMT material with TPL fractional order heat transfer at a constant temperature $T_{0}$, and with a magnetic field $\vec{H}_{0}=\left(0, H_{0}, 0\right)$ along $y$-axis. Furthermore, the Cartesian coordinates $x, y, z$ are considered with origin at $z=0$ towards $z$-axis with ramp heat directing vertically downwards.

For a 2-D model in the $x z$-plane, we take

$$
\mathbf{u}=(u, 0, w)
$$

Also, we consider that

$$
\mathbf{E}=0
$$




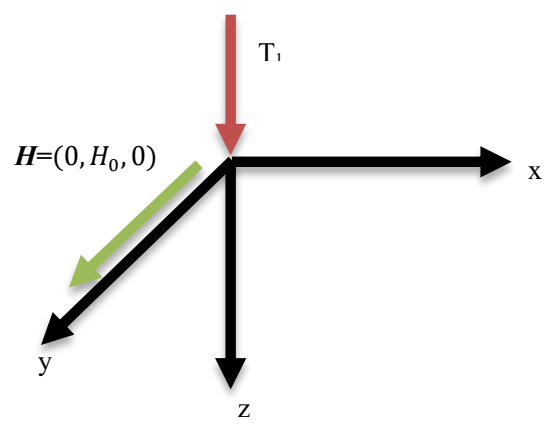

Fig. 1: Geometry of the problem.

And thus from the universal Ohm's law, we have

$$
J_{2}=0,
$$

$J_{1}$ and $J_{3}$ using (8) are given as

$$
\begin{aligned}
& J_{1}=\frac{\sigma_{0} \mu_{0} H_{0}}{1+m^{2}}\left(m \frac{\partial u}{\partial t}-\frac{\partial w}{\partial t}\right), \\
& J_{3}=\frac{\sigma_{0} \mu_{0} H_{0}}{1+m^{2}}\left(\frac{\partial u}{\partial t}+m \frac{\partial w}{\partial t}\right) .
\end{aligned}
$$

Following Slaughter [31] apply transformation on Eqs. (7 - (9) yeilds

(14) $c_{11} \frac{\partial^{2} u}{\partial x^{2}}+c_{13} \frac{\partial^{2} w}{\partial x \partial z}+c_{44}\left(\frac{\partial^{2} u}{\partial z^{2}}+\frac{\partial^{2} w}{\partial x \partial z}\right)-\beta_{1} \frac{\partial}{\partial x} T-\mu_{0} J_{3} H_{0}=\rho\left(\frac{\partial^{2} u}{\partial t^{2}}\right)$,

(15) $\left(c_{13}+c_{44}\right) \frac{\partial^{2} u}{\partial x \partial z}+c_{44} \frac{\partial^{2} w}{\partial x^{2}}+c_{33} \frac{\partial^{2} w}{\partial z^{2}}-\beta_{3} \frac{\partial}{\partial z} T-\mu_{0} J_{1} H_{0}=\rho\left(\frac{\partial^{2} w}{\partial t^{2}}\right)$,

(16) $K_{1}\left(1+\frac{\left(\tau_{t}\right)^{\alpha}}{\alpha !} \frac{\partial^{\alpha}}{\partial t^{\alpha}}\right) \frac{\partial^{2} \dot{T}}{\partial x^{2}}+K_{3}\left(1+\frac{\left(\tau_{t}\right)^{\alpha}}{\alpha !} \frac{\partial^{\alpha}}{\partial t^{\alpha}}\right) \frac{\partial^{2} \dot{T}}{\partial z^{2}}$

$$
\begin{aligned}
& +K_{1}^{*}\left(1+\frac{\left(\tau_{v}\right)^{\alpha}}{\alpha !} \frac{\partial^{\alpha}}{\partial t^{\alpha}}\right) \frac{\partial^{2} T}{\partial x^{2}}+K_{3}^{*}\left(1+\frac{\left(\tau_{v}\right)^{\alpha}}{\alpha !} \frac{\partial^{\alpha}}{\partial t^{\alpha}}\right) \frac{\partial^{2} T}{\partial z^{2}} \\
& =\left(1+\frac{\left(\tau_{q}\right)^{\alpha}}{\alpha !} \frac{\partial^{\alpha}}{\partial t^{\alpha}}+\frac{\left(\tau_{q}\right)^{2 \alpha}}{2 \alpha !} \frac{\partial^{2 \alpha}}{\partial t^{2 \alpha}}\right)\left[\rho C_{E} \ddot{T}+T_{0}\left\{\beta_{1} \frac{\partial \ddot{u}}{\partial x}+\beta_{1} \frac{\partial \ddot{w}}{\partial z}\right\}\right]
\end{aligned}
$$

and

$$
t_{x x}=c_{11} e_{x x}+c_{13} e_{x z}-\beta_{1} T,
$$




$$
\begin{aligned}
t_{z z} & =c_{13} e_{x x}+c_{33} e_{z z}-\beta_{3} T, \\
t_{x z} & =2 c_{44} e_{x z},
\end{aligned}
$$

where

$$
\beta_{1}=\left(c_{11}+c_{12}\right) \alpha_{1}+c_{13} \alpha_{3}, \quad \beta_{3}=2 c_{13} \alpha_{1}+c_{33} \alpha_{3} .
$$

Following dimensionless (DL) quantities are used to solve the problem:

$$
\begin{aligned}
& x^{\prime}=\frac{x}{L}, \quad u^{\prime}=\frac{\rho c_{1}^{2}}{L \beta_{1} T_{0}} u, \quad t^{\prime}=\frac{C_{1}}{L} t, \quad w^{\prime}=\frac{\rho c_{1}^{2}}{L \beta_{1} T_{0}} w, \\
& T^{\prime}=\frac{T}{T_{0}}, \quad t_{x x}^{\prime}=\frac{t_{x x}}{\beta_{1} T_{0}}, \quad t_{z z}^{\prime}=\frac{t_{z z}}{\beta_{1} T_{0}}, \quad t_{x z}^{\prime}=\frac{t_{x z}}{\beta_{1} T_{0}}, \\
& z^{\prime}=\frac{z}{L}, \quad \tau_{T}^{\prime}=\frac{C_{1}}{L} \tau_{T}, \quad \tau_{v}^{\prime}=\frac{C_{1}}{L} \tau_{v}, \quad \tau_{q}^{\prime}=\frac{C_{1}}{L} \tau_{q} .
\end{aligned}
$$

By using these DL quantities defined in (20) in the Eqs. (14 - (16), and thereafter suppressing primes, yields

$$
\frac{\partial^{2} u}{\partial x^{2}}+\delta_{1} \frac{\partial^{2} w}{\partial x \partial z}+\delta_{2} \frac{\partial^{2} u}{\partial z^{2}}-\frac{\partial T}{\partial x}=\frac{M}{1+m^{2}}\left[\frac{\partial u}{\partial t}+m \frac{\partial w}{\partial t}\right]+\frac{\partial^{2} u}{\partial t^{2}},
$$

$$
\delta_{1} \frac{\partial^{2} u}{\partial x \partial z}+\delta_{2} \frac{\partial^{2} w}{\partial x^{2}}+\delta_{3} \frac{\partial^{2} w}{\partial z^{2}}-\frac{\beta_{3}}{\beta_{1}} \frac{\partial T}{\partial z}=-\frac{M}{1+m^{2}}\left[m \frac{\partial u}{\partial t}-\frac{\partial w}{\partial t}\right]+\frac{\partial^{2} w}{\partial t^{2}}
$$

(23) $\left(1+\frac{C_{1}\left(\tau_{t}\right)^{\alpha}}{\alpha ! L} \frac{\partial^{\alpha+1}}{\partial t^{\alpha+1}}\right)\left(K_{1} \frac{\partial^{2} T}{\partial x^{2}}\right.$

$$
\begin{aligned}
& \left.+K_{3} \frac{\partial^{2} T}{\partial z^{2}}\right)+\left(1+\frac{\left(\tau_{v}\right)^{\alpha}}{\alpha !} \frac{\partial^{\alpha}}{\partial t^{\alpha}}\right)\left(K_{1}^{*} \frac{\partial^{2} T}{\partial x^{2}}+K_{3}^{*} \frac{\partial^{2} T}{\partial z^{2}}\right) \\
& =\left(1+\frac{\left(\tau_{q}\right)^{\alpha}}{\alpha !} \frac{\partial^{\alpha}}{\partial t^{\alpha}}+\frac{\left(\tau_{q}\right)^{2 \alpha}}{2 \alpha !} \frac{\partial^{2 \alpha}}{\partial t^{2 \alpha}}\right)\left[\rho C_{E} \ddot{T}+\frac{\beta_{1}}{\rho} T_{0}\left\{\beta_{1} \frac{\partial \ddot{u}}{\partial x}+\beta_{1} \frac{\partial \ddot{w}}{\partial z}\right\}\right],
\end{aligned}
$$

where

$$
\delta_{1}=\frac{c_{13}+c_{44}}{c_{11}}, \quad \delta_{2}=\frac{c_{44}}{c_{11}}, \quad \delta_{3}=\frac{c_{33}}{c_{11}}, \quad M=\left(\frac{L \sigma_{0} \mu_{0}^{2} H_{0}^{2}}{\rho C_{1}}\right) .
$$

According to initial and symmetry conditions

$$
\begin{aligned}
& \mathbf{u}(x, z, 0)=\dot{u}(x, z, 0)=0, \\
& \mathbf{w}(x, z, 0)=\dot{w}(x, z, 0)=0, \\
& \mathbf{T}(x, z, 0)=\dot{T}(x, z, 0)=0 .
\end{aligned}
$$


For $z \geq 0$ and $-\infty \leq x \leq \infty \mathbf{w}(x, z, t)=u(x, z, t)=\mathbf{T}(x, z, t)=0$, when $t>0$ while $z \rightarrow \infty$.

The Laplace and Fourier transforms are defined as

$$
\begin{aligned}
& \tilde{f}(x, z, s)=\int_{0}^{\infty} f(x, z, t) e^{-s t} d t, \\
& \hat{f}(\xi, z, s)=\int_{-\infty}^{\infty} \tilde{f}(x, z, s) e^{i \xi x} d x .
\end{aligned}
$$

On Eqs. (17) - (19) we get a set of equations

$$
\begin{aligned}
& \text { (26) } \begin{array}{r}
{\left[-\xi^{2}-s^{2}+\delta_{2} D^{2}-\frac{M s}{1+m^{2}}\right] \hat{u}(\xi, z, s)} \\
+\left[\delta_{1} D i \xi-\frac{m M s}{1+m^{2}}\right] \hat{w}(\xi, z, s)+(-\mathrm{i} \xi) \hat{T}(\xi, z, s)=0, \\
\text { (27) } \quad\left[\delta_{1} D i \xi+\frac{m M s}{1+m^{2}}\right] \hat{u}(\xi, z, s) \\
+\left[-\delta_{2} \xi^{2}+\delta_{3} D^{2}-s^{2}-\frac{M s}{1+m^{2}}\right] \hat{w}(\xi, z, s)-\frac{\beta_{3}}{\beta_{1}} D \hat{T}(\xi, z, s)=0,
\end{array}
\end{aligned}
$$

(28) $\frac{\beta_{1}^{2} T_{0} s^{2} i \xi}{\rho}\left[1+\frac{\tau_{q}^{\alpha} s^{\alpha}}{\alpha !}+\frac{\tau_{q}^{2 \alpha} s^{2 \alpha}}{2 \alpha !}\right] \hat{u}(\xi, z, s)$

$$
\begin{aligned}
& +\frac{\beta_{1} \beta_{3} T_{0} s^{2}}{\rho}\left[1+\frac{\tau_{q}^{\alpha} s^{\alpha}}{\alpha !}+\frac{\tau_{q}^{2 \alpha} s^{2 \alpha}}{2 \alpha !}\right] D \hat{w}(\xi, z, s) \\
& +\left\{\rho C_{E} C_{1}^{2} s^{2}\left(1+\frac{\tau_{q}^{\alpha} s^{\alpha}}{\alpha !}+\frac{\tau_{q}^{2 \alpha} s^{2 \alpha}}{2 \alpha !}\right)+K_{1} \xi^{2}\left(1+\frac{C_{1} \tau_{T}^{\alpha} s^{\alpha+1}}{\alpha ! L}\right)\right. \\
& +K_{1}^{*} \xi^{2}\left(1+\frac{\tau_{v}^{\alpha} s^{\alpha}}{\alpha ! L}\right)-\left[K_{3}\left(1+\frac{C_{1} \tau_{T}^{\alpha} s^{\alpha+1}}{\alpha ! L}\right)\right. \\
& \left.\left.+K_{3}^{*}\left(1+\frac{\tau_{v}^{\alpha} s^{\alpha}}{\alpha ! L}\right) D^{2}\right]\right\} \hat{T}(\xi, z, s)=0 .
\end{aligned}
$$

The non-trivial solution of (26) - (28) yields

$$
A D^{6}+B D^{4}+C D^{2}+E=0,
$$


where

$$
\begin{aligned}
D= & \frac{d}{d z}, \quad A=-\delta_{2} \delta_{3} A_{4}, \\
B= & -A_{4} A_{9} \delta_{2}+\delta_{2} \delta_{3} A_{3}-A_{5} A_{4} \delta_{3}-A_{2} A_{10} \delta_{2}+\delta_{1}^{2} A_{7}^{2} A_{4}, \\
C= & A_{3} A_{9} \delta_{2}-A_{9} A_{4} A_{5}+A_{5} A_{3} \delta_{3}-A_{2} A_{10} A_{5}+A_{8} A_{4} A_{6}-\delta_{1}^{2} A_{7}^{2} A_{3} \\
& +A_{7} A_{1} \delta_{1} A_{10}-\delta_{1} A_{7}^{2} A_{2}+A_{1} A_{7} \delta_{3}, \\
E= & A_{5} A_{9} A_{3}-A_{6} A_{8} A_{3}+A_{1} A_{9} A_{7}, \\
A_{1}= & \frac{\beta_{1}^{2} T_{0} s^{2} i \xi}{\rho}\left[1+\frac{\tau_{q}^{\alpha} s^{\alpha}}{\alpha !}+\frac{\tau_{q}^{2 \alpha} s^{2 \alpha}}{2 \alpha !}\right], \\
A_{2}= & \frac{\beta_{1} \beta_{3} T_{0} s^{2}}{\rho}\left[1+\frac{\tau_{q}^{\alpha} s^{\alpha}}{\alpha !}+\frac{\tau_{q}^{2 \alpha} s^{2 \alpha}}{2 \alpha !}\right], \\
A_{3}= & \rho C_{E} C_{1}^{2} s^{2}\left[1+\frac{\tau_{q}^{\alpha} s^{\alpha}}{\alpha !}+\frac{\tau_{q}^{2 \alpha} s^{2 \alpha}}{2 \alpha !}\right]+K_{1} \xi^{2}\left[1+\frac{C_{1} \tau_{T}^{\alpha} s^{\alpha+1}}{\alpha ! L}\right] \\
& +K_{1}^{*} \xi^{2}\left[1+\frac{\tau_{v}^{\alpha} s^{\alpha}}{\alpha ! L}\right], \quad A_{10}=-\frac{\beta_{3}}{\beta_{1}} . \\
A_{4}= & K_{3}\left[1+\frac{C_{1} \tau_{T}^{\alpha} s^{\alpha+1}}{\alpha ! L}\right]+K_{3}^{*}\left[1+\frac{\tau_{v}^{\alpha} s^{\alpha}}{\alpha ! L}\right], \\
A_{5}= & -\xi^{2}-s^{2}-\frac{M s}{1+m^{2}}, \quad A_{6}=-\frac{m M s}{1+m^{2}}, \\
A_{7}= & i \xi, \quad A_{8}=\frac{m M s}{1+m^{2}}, \quad A_{9}=-\xi^{2} \delta_{2}-s^{2}-\frac{M s}{1+m^{2}}, \quad A_{1}
\end{aligned}
$$

The $\pm \lambda_{j},(j=1,2,3)$ are the roots of Eq. (29). The result of Eqs. (26) - (28) satisfying the radiation condition, i.e. $\tilde{u}, \tilde{v}, \tilde{w} \rightarrow 0$ as $z \rightarrow \infty$ yields

$$
\begin{aligned}
\hat{u}(\xi, z, s) & =\sum_{j=1}^{3} B_{j} e^{-\lambda_{j} z}, \\
\hat{w}(\xi, z, s) & =\sum_{j=1}^{3} d_{j} B_{j} e^{-\lambda_{j} z}, \\
\hat{T}(\xi, z, s) & =\sum_{j=1}^{3} l_{j} B_{j} e^{-\lambda_{j} z},
\end{aligned}
$$


where $B_{j}, j=1,2,3$ being unknown constants and

$$
\begin{aligned}
& d_{j}=\frac{\delta_{2} A_{4} \lambda_{j}^{4}+\left(-A_{5} A_{4}+\delta_{2} A_{3}\right) \lambda_{j}^{2}+A_{1} A_{7}+A_{5} A_{3}}{-\delta_{3} \zeta_{4} \lambda_{j}^{4}+\left(\delta_{3} A_{3}-A_{2} A_{10}-A_{4} A_{9}\right) \lambda_{j}^{2}+A_{3} A_{6}}, \\
& l_{j}=\frac{\delta_{2} \delta_{3} \lambda_{j}^{4}+\left(\delta_{2} A_{9}+A_{5} \delta_{3}-\delta_{1}^{2} A_{7}^{2}\right) \lambda_{j}^{2}+A_{5} A_{9}-A_{6} A_{8}}{-\delta_{3} \zeta_{4} \lambda_{j}^{4}+\left(\delta_{3} A_{3}-A_{2} A_{10}-A_{4} A_{9}\right) \lambda_{j}^{2}+A_{3} A_{6}} .
\end{aligned}
$$

\section{BOUNDARY CONDITIONS}

The half-space surface $(z=0)$ is subjected to ramp type heat, therefore, we have

$$
\begin{aligned}
& t_{z z}(x, z, t)=0, \\
& t_{x z}(x, z, t)=0, \\
& T(x, z, t)=G(t) \delta(x),
\end{aligned}
$$

where $G(t)$ is a function defined as

$$
G(t)= \begin{cases}0 ; & t \leq 0 \\ T_{1} \frac{t}{t_{0}} ; & 0 \leq t \leq t_{0} \\ T_{1} ; & t>t_{0}\end{cases}
$$

Applying the transforms as defined by equations (24) - (25) to (35)

$$
\hat{T}(\xi, 0, s)=\bar{G}(s),
$$

where $\bar{G}(s)=T_{1}\left(1-e^{-s t_{0}}\right) / t_{0} s^{2}$.

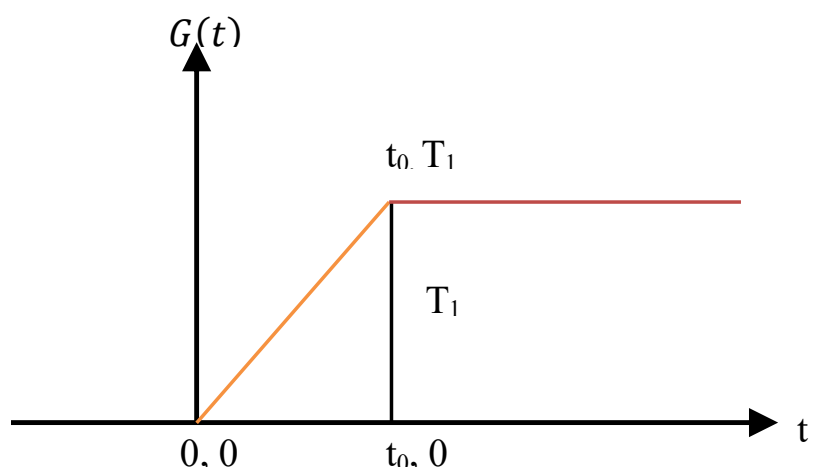

Fig. 2: Geometry of Ramp type heat. 
After using (24) - (25) respectively on (33) - (35) and (14) - (16) by using (30) - (32), we obtained the components of displacement, current density and stress, as well as conductive temperature

$$
\begin{aligned}
& {\left[\hat{u}, \hat{w}, \hat{T}, \hat{t}_{x z}, \hat{t}_{z z}, \hat{J}_{1}, \hat{J}_{3}\right](\xi, z, s)} \\
& =\frac{\bar{G}(s)}{\chi} \sum_{j=1}^{3}\left[1, d_{j}, l_{j}, \chi_{2 j}, \chi_{1 j}, \psi_{j}, \varrho_{j}\right] \varsigma_{j} e^{-\lambda_{j} z}
\end{aligned}
$$

where

$$
\chi_{1 j}=C_{13} i \xi-C_{33} d_{j} \lambda_{j}-\beta_{3} l_{j}, \quad \chi_{2 j}=\left(\lambda_{j}+i \xi d_{j}\right), \quad \chi_{3 j}=l_{j}, \quad j=1,2,3
$$

and

$$
\begin{array}{ll}
\chi=\varsigma_{1} \chi_{11}-\varsigma_{2} \chi_{12}+\varsigma_{3} \chi_{13}, & \varsigma_{1}=\bar{G}(s)\left(\chi_{12} \chi_{23}-\chi_{13} \chi_{22}\right), \\
\varsigma_{2}=\bar{G}(s)\left(\chi_{11} \chi_{23}-\chi_{21} \chi_{13}\right), & \varsigma_{3}=\bar{G}(s)\left(\chi_{11} \chi_{23}-\chi_{21} \chi_{12}\right), \\
\psi_{j}=\frac{\sigma_{0} H_{0} \mu_{0}}{\left(1+m^{2}\right)} \mathrm{s}\left(m-d_{j}\right), & \varrho_{j}=\frac{\sigma_{0} H_{0} \mu_{0}}{\left(1+m^{2}\right)} \mathrm{s}\left(1+m d_{j}\right) .
\end{array}
$$

\section{INVERSION OF THE TRANSFORMATION}

To obtain the results in physical domain, Fourier transform in Eq. (36) need be inverted by using

$$
\tilde{f}(x, z, s)=\frac{1}{2 \pi} \int_{-\infty}^{\infty} e^{-i \xi x} \hat{f}(\xi, z, s) d \xi=\frac{1}{2 \pi} \int_{-\infty}^{\infty}\left|\cos (\xi x) f_{e}-i \sin (\xi x) f_{0}\right| d \xi
$$

The Laplace transform function $\tilde{f}(x, z, s)$ following Honig and Hirdes [32], inverted to $f(x, z, t)$ by

$$
f(x, z, t)=\frac{1}{2 \pi i} \int_{\nu-i \infty}^{\nu+i \infty} \tilde{f}(x, z, s) e^{-s t} d s .
$$

Romberg's integration [33] is used for inversion integral in Eq. (37).

\section{Numerical Results AND Discussion}

The physical data for cobalt material [34] is used to validate the theoretic outcomes and influence of fractional order parameter and Hall current, is given as

$$
c_{11}=3.07 \times 10^{11} \mathrm{Nm}^{-2}, \quad \mathrm{~T}_{0}=298 \mathrm{~K}, \quad c_{44}=1.510 \times 10^{11} \mathrm{Nm}^{-2},
$$




$$
\begin{gathered}
\varepsilon_{0}=8.838 \times 10^{-12} \mathrm{Fm}^{-1}, \quad \beta_{1}=7.04 \times 10^{6} \mathrm{Nm}^{-2} \mathrm{deg}^{-1}, \\
\beta_{3}=6.90 \times 10^{6} \mathrm{Nm}^{-2} \mathrm{deg}^{-1}, c_{33}=3.581 \times 10^{11} \mathrm{Nm}^{-2}, \quad \rho=8.836 \times 10^{3} \mathrm{kgm}^{-3}, \\
C_{E}=4.27 \times 10^{2} \mathrm{Jkg}^{-1} \mathrm{deg}^{-1}, \quad \mathrm{H}_{0}=1 \mathrm{Jm}^{-1} \mathrm{nb}^{-1}, \quad K_{1}=0.690 \times 10^{2} \mathrm{Wm}^{-1} \mathrm{~K}^{-1}, \\
c_{13}=1.027 \times 10^{10} \mathrm{Nm}^{-2}, \quad K_{3}=0.690 \times 10^{2} \mathrm{Wm}^{-1} \mathrm{~K}^{-1}, \quad L=1 .
\end{gathered}
$$

Dimensionless field variables are compared with distinct values of fractional order factor and constant Hall current factor $m=0.5$ is shown graphically. Figure 3 and Fig. 4 exhibit the variations in $u$ and $w$ w.r.t $x$ respectively. For different values of $\alpha$ the displacement variables $u$ and $w$, first decreases when $0 \leq x \leq 2$, and thereafter exhibits oscillatory pattern.

Figure 5 shows the variations in $t_{z x}$ w.r.t. $x$ and Fig. 6 illustrates the $t_{z z}$ w.r.t. $x$. For $\alpha=1.0$ the tangential stress $t_{z x}$ shows an oscillatory behaviour. However, when $\alpha=0.5, t_{z x}$ and $t_{z z}$ shows an oscillatory behaviour with declining amplitude with rise in $x$ and the normal stress $t_{z z}$ shows oscillatory behaviour with larger amplitude with increase in $x$. For $\alpha=1.5, t_{z x}$ abruptly increases when $0 \leq x \leq 2$ and then become oscillatory while normal stress $t_{z z}$ abruptly rises when $0 \leq x \leq 2$ and then shows oscillatory behaviour with declining amplitude with rise in $x$. Figure 7 shows the variations in $T$ with $x$. Initially, it sharply decrease and then become oscillatory for rest of the length. Figure 8 illustrates the $J_{1}$ and Fig. 9 demonstrates the $J_{3}$ w.r.t.

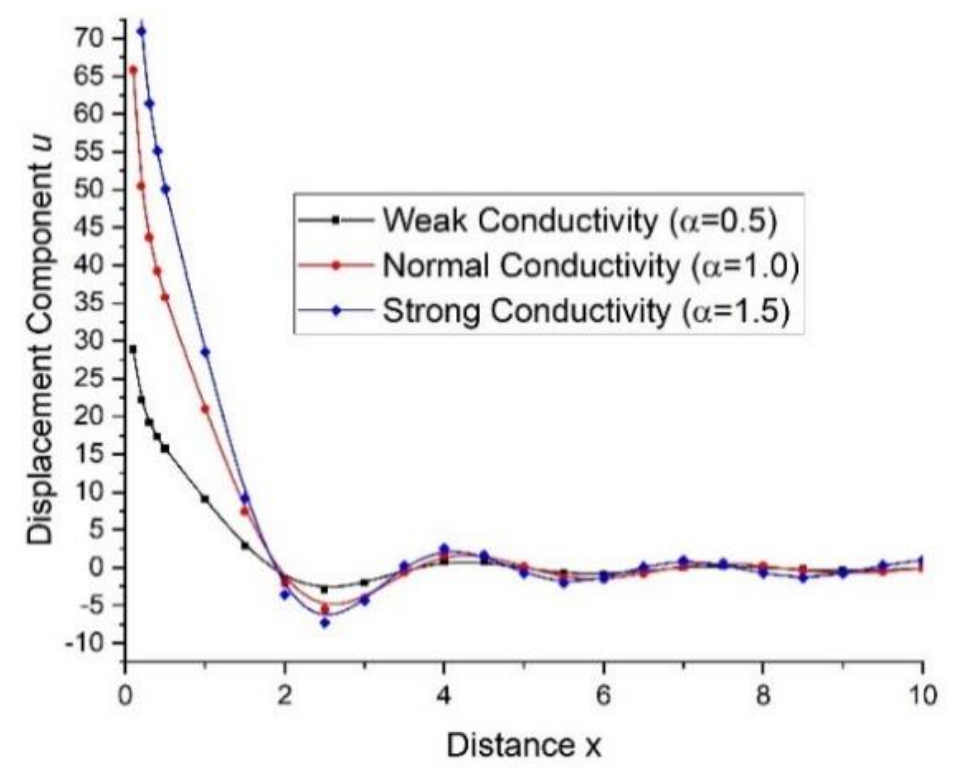

Fig. 3: Deviations of $u$ w.r.t. $x$. 


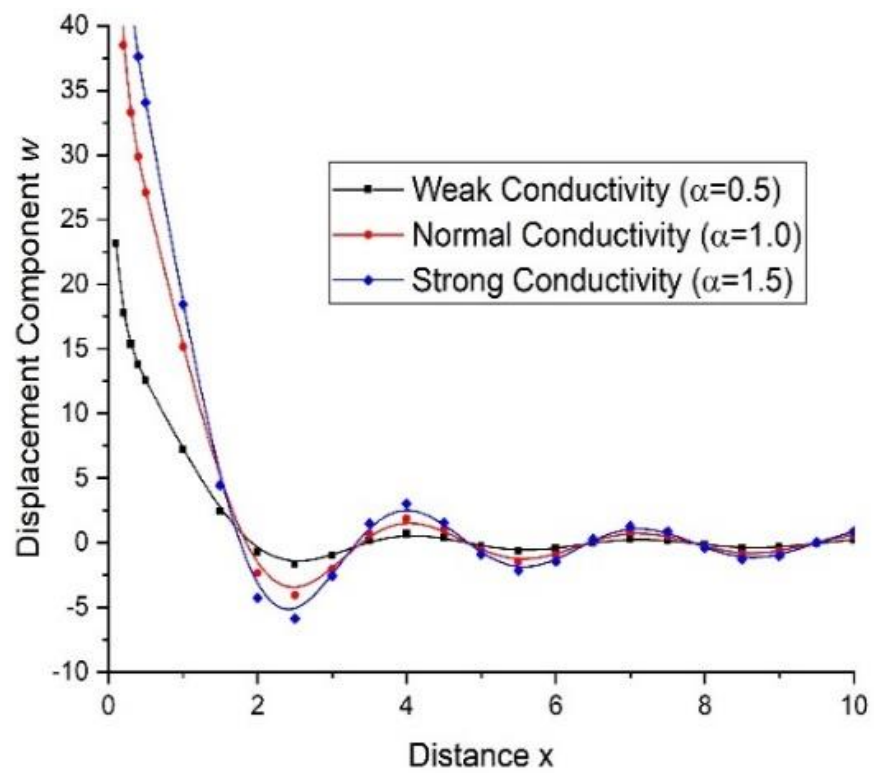

Fig. 4: Variations of $w$ w.r.t. $x$.

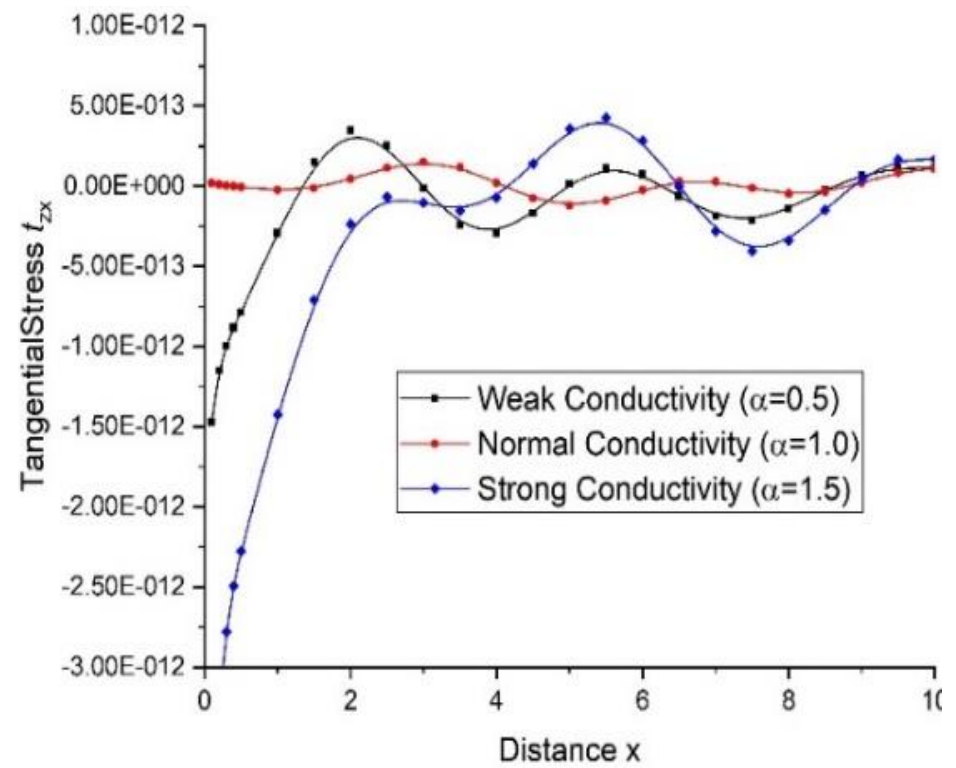

Fig. 5: Deviations of $t_{z x}$ w.r.t. $x$. 


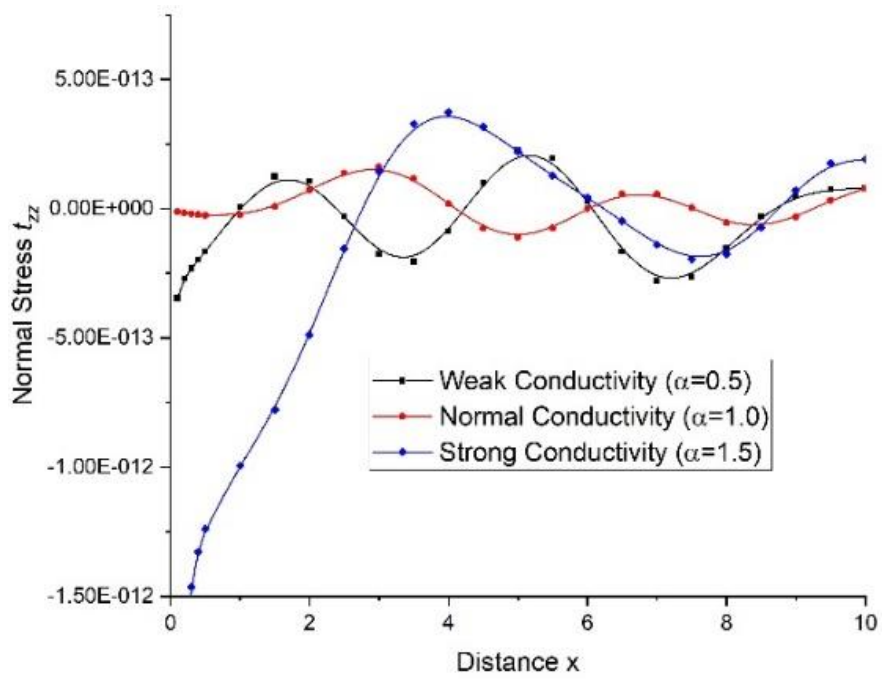

Fig. 6: Deviations of $t_{z z}$ w.r.t. $x$.

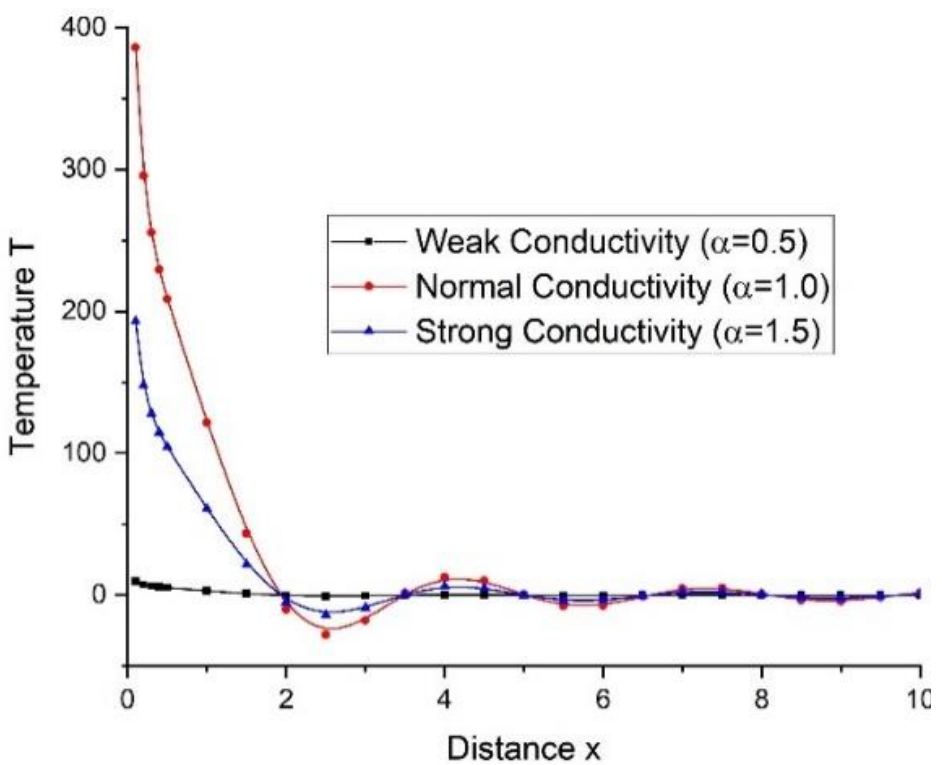

Fig. 7: Deviations of $T$ with $x$.

$x$. for $\alpha=0.5$ and $\alpha=1.0$ the $J_{1}$ is remain similar, while $J_{3}$ for $\alpha=0.5$ the value remains similar and for $\alpha=1.0$ the value of of $J_{3}$ first increases when $0 \leq x \leq 2$ and then remains identical as for $\alpha=0.5$. 
234 Fractional Order Theory of Thermal Stresses in a $2 D$ Transversely ...

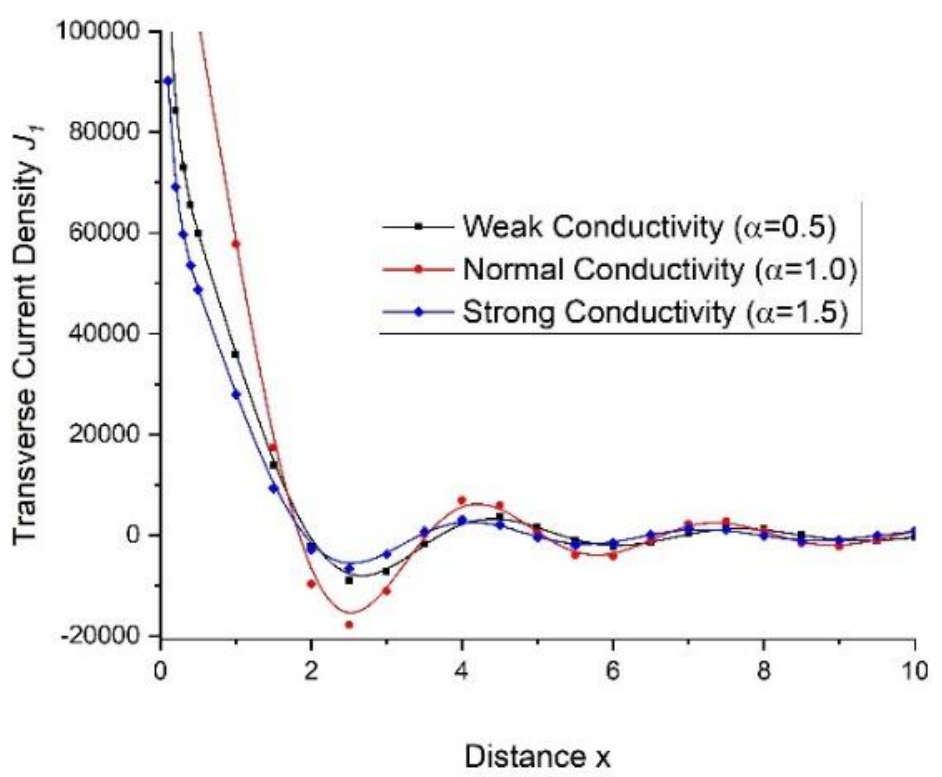

Fig. 8: Deviations of $J_{1}$ w.r.t. $x$.

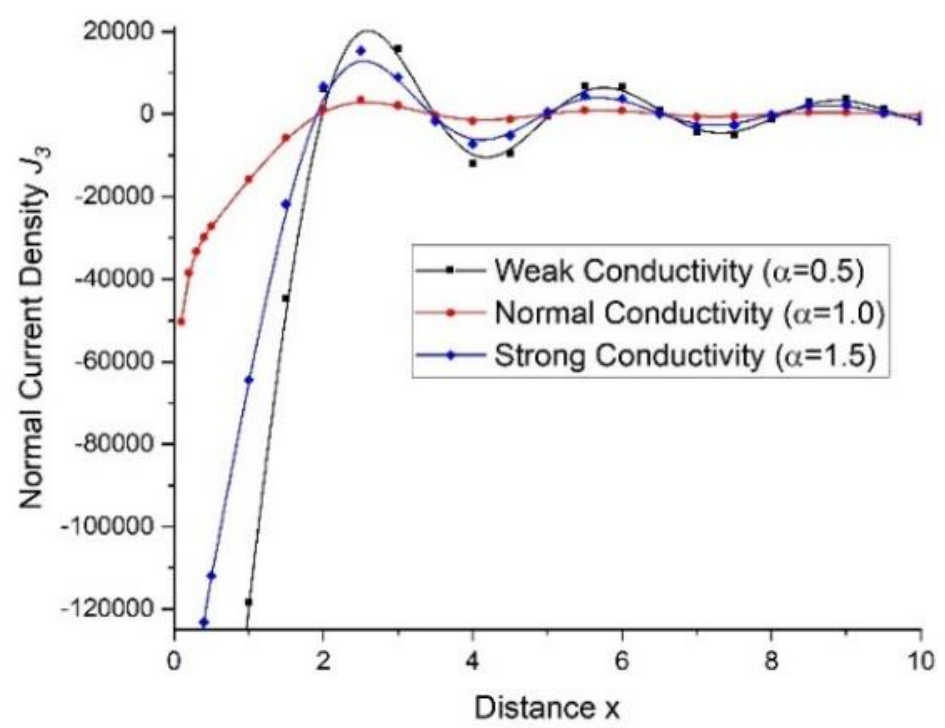

Fig. 9: Deviations of $J_{3}$ w.r.t. $x$. 


\section{CONCLUSION}

- A novel mathematical model is purposed for the study of HTIMT material with FOT of thermal stresses due to ramp type heat and Hall current effect

- Fractional order parameter $\alpha$ has a substantial impact on the various parameters of HTIMT medium. As the value of $\alpha$ increases, it shows the major effect on the various components.

- The effect of ramp heat in HTIMT medium with Hall Effect and fractional order factor plays a significant role in the analysis of the deformed media.

- The graphs shows the effect of $\alpha$ with constant value of $m$ on the media and also fulfil research motive.

\section{REFERENCES}

[1] H.H. Sherief, A.M.A. El-Sayed, A. El-Latief (2010) Fractional Order Theory of Thermoelasticity. International Journal of Solids and Structures 47(2) 269-275.

[2] H. Youssef (2006) Two-Dimensional Generalized Thermoelasticity Problem For A Half Space Subjected To Ramp - Type Heating. European Journal of Mechanics A/Solid 25 745-763.

[3] H. Youssef (2010) Theory of Fractional Order Generalized Thermoelasticity. ASME J. Heat Transfer 132 1-7.

[4] M.A. EzZAT, A.S. KARAmany, M.A. FAYIK (2011) Fractional Order Theory in Thermoelastic Solid with Three-Phase Lag Heat Transfer. Archive of Applied Mechanics 82 557-572.

[5] M.A. EzZat, A.S. El-Karamany, S.M. EzZat (2012) Two-Temperature Theory in Magneto-Thermoelasticity with Fractional Order Dual-Phase-Lag Heat Transfer. $\mathrm{Nu}$ clear Engineering and Design (Elsevier) 252 267-277.

[6] M.A. EzZat, A.S. El-Karamany, A.A. El-BARY (2017) Two-Temperature Theory in Green-Naghdi Thermoelasticity with Fractional Phase-Lag Heat Transfer. Microsystem Technologies - Springer Nature 24(2) 951-961.

[7] M. BAChHer, N. SARKAR (2016) Fractional Order Magneto-Thermoelasticity in a Rotating Media with One Relaxation Time. Mathematical Models In Engineering 2(1) 56-68.

[8] R. Kumar, K. Singh (2015) Interaction of Fractional Order Theories in Micropolar Thermoelastic Solid Due to Ramp Type Heating. Proceedings of The World Congress on Engineering I. London, U.K.

[9] R. Kumar, N. Sharma, P. Lata (2016) Thermomechanical Interactions Due to Hall Current in Transversely Isotropic Thermoelastic with and without Energy Dissipation with Two Temperatures and Rotation. Journal of Solid Mechanics 8(4) 840-858. 
[10] S.S. Sheoran, P. Kundu (2016) Fractional Order Generalized Thermoelasticity Theories: A Review. International Journal of Advances in Applied Mathematics and Mechanics 3(4) 76-81.

[11] I. AbBas (2018) A Study on Fractional Order Theory in Thermoelastic Half-Space under Thermal Loading. Physical Mesomechanics 21(2) 150-156.

[12] M. MARIN (1997) Cesaro Means in Thermoelasticity of Dipolar Bodies. Acta Mechanica 122(1-4) 155-168.

[13] M. MARIN (1994) The Lagrange Identity Method in Thermoelasticity of Bodies with Microstructure. International Journal of Engineering Science 32(8) 1229-1240. DOI: 10.1016/0020-7225(94)90034-5.

[14] M. Marin, R. Agarwal, L. Codarcea (2017) A Mathematical Model For ThreePhase-Lag Dipolar Thermo-Elastic Bodies. Journal of Iinequalities and Application 2017(1) 1-16. DOI: 10.1186/S13660-017-1380-5.

[15] M. Marin, E. Craciun, N. Pop (2016) Considerations On Mixed Initial-Boundary Value Problems for Micropolar Porous Bodies. Dynamic Systems and Applications 25(1-2) 175-196.

[16] P. LATA, I. KAUR (2018) Effect of Hall Current in Transversely Isotropic Magnetothermoelastic Rotating Medium with Fractional Order Heat Transfer Due to Normal Force. Advances in Materials Research 7(3) 203-220.

[17] P. LAtA, I. KaUR (2019) Transversely Isotropic Thermoelastic Thin Circular Plate with Time Harmonic Sources. Geomechanics and Engineering 19(1) 29-36. DOI: 10.12989/gae.2019.19.1.029.

[18] M. MARIN (2009) On the Minimum Principle for Dipolar Materials with Stretch. Nonlinear Analysis Real World Applications 10(3) 1572-1578.

[19] M. MARIN (2010) Lagrange Identity Method for Microstretch Thermoelastic Materials. J. of Mathematical Analysis and Applications 363(1) 275-286.

[20] R. Kumar, P. Kaushal, R. Sharma (2018) Transversely Isotropic Magneto-Visco Thermoelastic Medium with Vacuum and Without Energy Dissipation. Journal of Solid Mechanics 10(2) 416-434.

[21] P. LATA, I. KaUR (2019) Axisymmetric Thermomechanical Analysis of Transversely Isotropic Magneto Thermoelastic Solid Due to Time-Harmonic Sources. Coupled Systems Mechanics 8(5) 415-437. DOI: 10.12989/csm.2019.8.5.415.

[22] P. LATA, I. KAUR (2019) Plane Wave Propagation in Transversely Isotropic Magnetothermoelastic Rotating Medium with Fractional Order Generalized Heat Transfer. Structural Monitoring and Maintenance 6(3) 191-218. DOI: 10.12989/smm.2019.6.3.191.

[23] P. LATA, I. KAUR (2019) Effect of Rotation and Inclined Load on Transversely Isotropic Magneto Thermoelastic Solid. Structural Engineering And Mechanics 70(2) 245-255.

[24] P. LATA, I. KaUR (2019) Thermomechanical Interactions in Transversely Isotropic Magneto Thermoelastic Solid with Two Temperatures and without Energy Dissipation. Steel and Composite Structures 32(6) 779-793. DOI: 10.12989/scs.2019.32.6.779. 
[25] P. LATA, I. KAUR (2019) Transversely Isotropic Magneto Thermoelastic Solid With Two Temperature and Without Energy Dissipation in Generalized Thermoelasticity Due to Inclined Load. Sn Applied Sciences 1 426. DOI: 10.1007/s42452-019-0438-z.

[26] P. LATA, I. Kaur (2019) Thermomechanical Interactions Due to Time Harmonic Sources in a Transversely Isotropic Magneto Thermoelastic Solids with Rotation. International Journal of Microstructure and Materials Properties 14(6) 549-577.

[27] M. MARIN, E.CraCiUn (2017) Uniqueness Results For A Boundary Value Problem In Dipolar Thermoelasticity To Model Composite Materials. Composites Part B: Engineering 126 27-37.

[28] I. KaUR, P. LATA (2019) Rayleigh Wave Propagation in Transversely Isotropic Magneto Thermoelastic Medium with Three Phase Lag Heat Transfer and Diffusion. International Journal of Mechanical and Materials Engineering 14(12) 1-11. DOI: 10.1186/s40712-019-0108-3.

[29] R. Kumar, N. Sharma, P. LATA (2017) Effects of Hall Current and Two Temperatures Intransversely Isotropic Magnetothermoelastic with and without Energy Dissipation Due to Ramp-Type Heat. Mechanics of Advanced Materials and Structures 24(8) 625-635.

[30] M. Schoenberg, D. Censor (1973) Elastic Waves in Rotating Media. Quarterly of Applied Mathematics 31 115-125.

[31] W. Slaughter (2002) "The Linearised Theory of Elasticity". Birkhausar.

[32] G. Honig, U. Hirdes (1984) A Method for the Numerical Inversion of Laplace Transform. Journal of Computational and Applied Mathematics 10 113-132.

[33] W. Press, S.A. Teukolshy W.T. Vellerling, B. Flannery (1986) "Numerical Recipes in Fortran". Cambridge University Press, Cambridge.

[34] R. Dhaliwal, A. Singh (1980) "Dynamic Coupled Thermoelasticity". New DelhiIndia: Hindustan Publication Corporation. 